\title{
Reply to "Questions About In-Breast Tumor Recurrence in Patients Treated with Breast-Conserving Therapy"
}

\author{
James Laird, $\mathrm{BA}^{1}$ and Lior Z. Braunstein, $\mathrm{MD}^{2}$ \\ ${ }^{1}$ New York University School of Medicine, New York, NY; ${ }^{2}$ Department of Radiation Oncology, Memorial Sloan- \\ Kettering Cancer Center, New York, NY
}

Dr. Altundag raises an interesting question regarding the influence of breast cancer biologic subtype on survival after a local recurrence. ${ }^{1}$ Although we previously reported that subtype approximations do maintain prognostic significance after a first recurrence, ${ }^{2}$ in the context of the current study, this raises another issue about the propensity for a breast cancer recurrence to harbor ductal carcinoma in situ (DCIS) as a function of the primary disease subtype. This was addressed in Table 2, demonstrating no significant differences in the rates between true recurrence (TR) and new primaries (NP) by biologic subtype.

In addition, with regard to the overall cohort from which these recurrences derive, the nearly 4000 patients treated with breast-conserving therapy (BCT) were representative of previously reported American population distributions of breast cancer biologic subtypes, including approximately $80 \%$ estrogen receptor-positive $(\mathrm{ER}+)$ lesions, the majority of which retained their luminal (ER+) subtype at the time of local recurrence. We currently are performing additional analyses to identify whether variations in subtype and location between primary and recurrent lesions are as informative as our DCIS definition of true recurrence versus new primary disease.

Sincerely,

James Laird, BA and Lior Z. Braunstein, MD

\section{REFERENCE}

1. Altundag K (2017) Questions about in-breast tumor recurrence in patients treated with breast-conserving therapy. Ann Surg Oncol. https://doi.org/10.1245/s10434-017-6282-8

2. Braunstein LZ et al (2015) Outcome following local-regional recurrence in women with early-stage breast cancer: impact of biologic subtype. Breast J. 21(2):161-167 